\title{
Options on realized variance in Log-OU models
}

\author{
Drimus, Gabriel G
}

\begin{abstract}
We study the pricing of options on realized variance in a general class of Log-OU stochastic volatility models. The class includes several important models proposed in the literature. Having as common feature the log-normal law of instantaneous variance, the application of standard Fourier-Laplace transform methods is not feasible. We derive extensions of Asian pricing methods, to obtain bounds, in particular, a very tight lower bound for options on realized variance.
\end{abstract}

DOI: https://doi.org/10.1080/1350486X.2011.639951

Posted at the Zurich Open Repository and Archive, University of Zurich

ZORA URL: https://doi.org/10.5167/uzh-55645

Journal Article

Accepted Version

Originally published at:

Drimus, Gabriel G (2012). Options on realized variance in Log-OU models. Applied Mathematical Finance, 19(5):477-494.

DOI: https://doi.org/10.1080/1350486X.2011.639951 


\title{
Options on realized variance in Log-OU models
}

\author{
Gabriel G. Drimus * \\ Department of Mathematics \\ University of Copenhagen \\ Universitetsparken 5, DK-2100 \\ Denmark \\ Previous version: April, 2011 \\ Current version: October, 2011
}

\begin{abstract}
We study the pricing of options on realized variance in a general class of Log-OU stochastic volatility models. The class includes several important models proposed in the literature. Having as common feature the log-normal law of instantaneous variance, the application of standard Fourier-Laplace transform methods is not feasible. We derive extensions of Asian pricing methods, to obtain bounds, in particular, a very tight lower bound for options on realized variance.
\end{abstract}

KEYWORDS: options on realized variance, Asian options, stochastic volatility.

JEL: C63, G13.

\section{Introduction}

In this paper, we work in a general class of Log-OU stochastic volatility models; this framework includes, for example, Scott's (1987) model and the continuous version of Bergomi's (2005) model. A desirable property of this class of models is that the distribution of realized variance will be approximately log-normal. Several authors including Bergomi (2005) and Gatheral (2006) have found that, in practice, the distribution of realized variance is close to log-normal.

Despite its numerical tractability, the standard Heston (1993) model has the major drawback that it generates a downward sloping volatility-ofvolatility skew, a feature which is strongly at odds with variance markets in

*gdrimus@math.ku.dk 
practice. The class of Log-OU models discussed in this paper will generate an approximately flat, in fact mildly upward sloping, volatility-of-volatility skew thus providing a significant improvement over the standard Heston (1993) model.

The class of models considered have the common property that the marginal distribution of instantaneous variance is log-normal. By the wellknown fact that a sum of log-normal variables does not remain log-normal, the realized variance - defined as an integral over the instantaneous variances - will not be exactly log-normal and, more importantly, will not have a moment generating function (or Laplace transform) in closed-form. Therefore familiar Fourier-Laplace transform methods cannot be applied to value options on realized variance. Motivated by the similarities between options on realized variance and Asian options, we extend the classical Asian bounds of Rogers, Shi (1995) and Thompson (1999) to Log-OU processes. In particular, we obtain a very tight lower bound, which can essentially be used as the true price.

The remaining of this paper is organized as follows. In the next section we introduce the class of Log-OU models and discuss some of their general properties. The following section develops the bounds for options on realized variance and illustrates their numerical performance. The fourth part discusses further applications and shows how the results can be applied to accommodate multiple factors and discrete sampling of realized variance. The final section summarizes the conclusions.

\section{A class of Log-OU Models}

We start by specifying the Log-OU stochastic volatility dynamics considered throughout the paper. Let $\left(B_{t}, W_{t}\right)_{t \geq 0}$ be a standard two-dimensional Brownian motion defined on a filtered probability space $\left(\Omega, \mathcal{F}, \mathcal{F}_{t}, \mathbb{Q}\right)$ satisfying the usual conditions. We assume that the stock price and its instantaneous variance $\left(S_{t}, v_{t}\right)_{t \geq 0}$ satisfy the following dynamics under the risk neutral measure $\mathbb{Q}$ :

$$
\begin{aligned}
\frac{d S_{t}}{S_{t}} & =(r-\delta) d t+\sqrt{v_{t}}\left(\rho d W_{t}+\sqrt{1-\rho^{2}} d B_{t}\right) \\
d \log \left(v_{t}\right) & =\left[k\left(\log (\theta(t))-\log \left(v_{t}\right)\right)+\chi(t)\right] d t+\epsilon d W_{t}
\end{aligned}
$$

where $\theta(t)$ and $\chi(t)$ are arbitrary deterministic functions. The risk free interest rate, dividend yield and correlation parameters are denoted $r, \delta$ and $\rho$ respectively. We recognize that the logarithm of the instantaneous variance $y_{t}=\log \left(v_{t}\right)$ follows a Gaussian Ornstein- ̈̈hlenbeck process with extra drift term $\chi(t)$.

The simplest special case of (2) is obtained for $\theta(t)=\theta$ (a constant) and $\chi(t)=0$, which yields the classical model of Scott (1987). More recently, we 
find in Bergomi (2005) an interesting variation where one starts by specifying the dynamics of the instantaneous forward variances $\xi_{t}^{T}=E^{\mathbb{Q}}\left(v_{T} \mid \mathcal{F}_{t}\right)$, similar to HJM forward interest rate modeling, as follows:

$$
d \xi_{t}^{T}=\epsilon \cdot \xi_{t}^{T} \cdot e^{-k(T-t)} d W_{t}
$$

along with an initial instantaneous forward variance curve $\xi_{0}^{T}$, for all $T>0$. We now show that (3) is also a Log-OU model. Assuming the initial forward variance curve is sufficiently smooth (more precisely, differentiable in $T$ ), we start by defining the function $\theta(\cdot)$ as:

$$
\log (\theta(T))=\log \xi_{0}^{T}+\frac{1}{k} \cdot \frac{\partial \log \xi_{0}^{T}}{\partial T} .
$$

A straightforward application of Itô's lemma to the logarithm of the instantaneous variance $y_{t}=\log \left(\xi_{t}^{t}\right)$ reveals the dynamics:

$$
d y_{t}=\left[k\left(\log (\theta(t))-y_{t}\right)-\frac{\epsilon^{2}}{4}\left(1+e^{-2 k t}\right)\right] d t+\epsilon d W_{t}
$$

which we recognize as a special case of $(2)$ with $\chi(t)=-\frac{\epsilon^{2}}{4}\left(1+e^{-2 k t}\right)$. This parametrization may appear less intuitive, but has the advantage that it provides a perfect fit to any initial (forward) variance swap curve and it simplifies the dynamics of forward variances as in (3).

In what follows, we will find it convenient to write the logarithm of the instantaneous variance $y_{t}$ as $y_{t}=\bar{y}(t)+Z_{t}$ where $\bar{y}(t)$ is the (time dependent) mean of $y_{t}$ and $Z_{t}$ is a centered Gaussian-OU process given by

$$
Z_{t}=\epsilon \cdot e^{-k t} \cdot \int_{0}^{t} e^{k s} d W_{s}
$$

Applying Itô's lemma to $e^{k t} \cdot y_{t}$ and using the Log-OU dynamics (2) we obtain, for the deterministic component $\bar{y}(t)$ :

$$
\bar{y}(t)=y_{0} e^{-k t}+k e^{-k t} \int_{0}^{t} e^{k u} \log (\theta(u)) d u+e^{-k t} \int_{0}^{t} e^{k u} \chi(u) d u .
$$

For Scott's (1987) model, the expression (6) simplifies to

$$
\bar{y}(t)=\log (\theta)+\left(y_{0}-\log (\theta)\right) e^{-k t}
$$

and for Bergomi's (2005) model we have

$$
\bar{y}(t)=y_{0} e^{-k t}+k e^{-k t} \int_{0}^{t} e^{k u} \log (\theta(u)) d u-\frac{\epsilon^{2}}{4 k}\left(1-e^{-2 k t}\right) .
$$

In Log-OU stochastic volatility models of the form (1)-(2), there is no fast analytical method to compute prices of European vanilla options. Nevertheless, efficient one-dimensional Monte Carlo pricing can be obtained by 


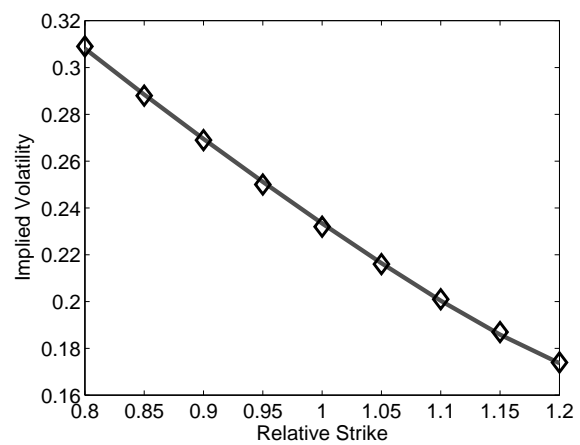

Figure 1: Fit of Scott (1987) model to 6M implied volatilities of S\&P500 index options on December 14, 2009. Diamond Black: Market implied volatilities. Solid grey: Scott's model implied volatility curve. Parameters obtained are: $v_{0}=19.85 \%^{2}, k=1.786, \theta=26.32 \%^{2}, \epsilon=2.19, \rho=-0.84$.

using the so-called "mixing" approach (see Romano, Touzi (1997) or Lewis $(2000))$. We start with a simple application of Itô's lemma to $\log \left(S_{t}\right)$ on the interval $[0, T]$ to obtain:

$$
\begin{aligned}
S_{T}= & S_{0} \cdot \exp \left(-\frac{1}{2} \rho^{2} \int_{0}^{T} e^{y_{t}} d t+\rho \int_{0}^{T} \sqrt{e^{y_{t}}} d W_{t}\right) \\
& \cdot \exp \left((r-\delta) T-\frac{1}{2}\left(1-\rho^{2}\right) \int_{0}^{T} e^{y_{t}} d t+\sqrt{1-\rho^{2}} \int_{0}^{T} \sqrt{e^{y_{t}}} d B_{t}\right) .
\end{aligned}
$$

By conditioning on the path of the Brownian Motion $W_{t}$, driving the instantaneous variance, we obtain the price of any European option as an expectation over Black-Scholes prices. If we let $C^{B S}(S, \sigma)$ denote the BlackScholes price of a European vanilla option, for initial spot $S$ and constant volatility $\sigma$, we arrive at the following mixing representation for option prices in our stochastic volatility models:

$$
C^{S V}=E^{\mathbb{Q}}\left(C^{B S}\left(S_{0} \cdot \xi_{T}, \sqrt{1-\rho^{2}} \sqrt{\frac{1}{T} \int_{0}^{T} e^{y_{t}} d t}\right)\right)
$$

where

$$
\xi_{T}=\exp \left(-\frac{1}{2} \rho^{2} \int_{0}^{T} e^{y_{t}} d t+\rho \int_{0}^{T} \sqrt{e^{y_{t}}} d W_{t}\right)
$$

To obtain a set of parameter values for our subsequent numerical examples, we end this section with a simple fit of the Scott (1987) model to market prices of 6-months S\&P500 index options on Dec 14, 2009; figure (1) displays the fit. The parameters obtained are : $v_{0}=19.85 \%^{2}, k=1.786$, $\theta=26.32 \%^{2}, \epsilon=2.19, \rho=-0.84$. We shall use this example set of parameters to illustrate the numerical results in the next section. 


\section{Options on realized variance in Log-OU models}

We now consider the problem of pricing options on realized variance in the general class of Log-OU stochastic volatility models (1)-(2). The payoff of a call option on realized variance, with strike $K$ and maturity $T$, is given by:

$$
\left(\frac{1}{T} \int_{0}^{T} v_{t} d t-K\right)_{+}
$$

In models, such as Heston (1993), where the Fourier-Laplace transform $E^{\mathbb{Q}}\left(\exp \left(-s \cdot \frac{1}{T} \int_{0}^{T} v_{t} d t\right)\right)$ with $s \in \mathbb{C}$ and $\operatorname{Re}(s)>s^{*} \geq 0$ is known in closed form, semi-analytical transform techniques can be applied to value options on realized variance (see, for example, Sepp (2008)). In the class of Log-OU models the transform is not available in closed form.

In what follows, we shall regard the payoff (7) as the payoff of an Asian option written on the instantaneous variance process $v_{t}$. Of course, a direct valuation approach is to construct a Monte Carlo scheme. Similar to arithmetic average Asian stock options in Kemma, Vorst (1990), we can implement a Monte Carlo approach, enhanced with geometric average control variates. Specifically, recalling that $v_{t}=\exp \left(\bar{y}(t)+Z_{t}\right)$, in order to value

$$
C_{v a r}(K)=e^{-r T} E^{\mathbb{Q}}\left(\frac{1}{T} \int_{0}^{T} e^{\bar{y}(t)+Z_{t}} d t-K\right)_{+}
$$

we can use the control variate

$$
C_{\text {var }}^{\text {Geom. }}(K)=e^{-r T} E^{\mathbb{Q}}\left(\exp \left(\frac{1}{T} \int_{0}^{T}\left(\bar{y}(t)+Z_{t}\right) d t\right)-K\right)_{+} .
$$

The latter can be valued with a Black-Scholes style formula as provided in Appendix A.1.

Subsequently, Rogers, Shi (1995) and then Thompson (1999), developed elegant methods to bound the prices of arithmetic average Asian options. In particular, they discovered an extremely tight lower bound, which follows from a simple conditioning argument. Throughout the Asian pricing literature, the log-stock price was assumed in the classical Black-Scholes model:

$$
\log \left(S_{t}\right)=\log \left(S_{0}\right)+\left(r-\delta-\frac{\sigma^{2}}{2}\right) t+\sigma Z_{t}
$$

where $Z_{t}$ is a standard Brownian motion. Note that, for our "Asian" option, $\log \left(v_{t}\right)$ has a similar form, namely $\log \left(v_{t}\right)=\bar{y}(t)+Z_{t}$, except in this case, $Z_{t}$ is a mean-reverting Ornstein-Ühlenbeck process. It is not clear that the classical Asian methods can be extended to this setting. We next show how to extend the Asian bounds to a mean-reverting process $Z_{t}$ and obtain, in 
particular, a very tight lower bound for options on realized variance in the general class of Log-OU stochastic volatility models. The lower bound is so accurate that, in practice, it can be used as a substitute for the true price.

The basic idea rests on the following straightforward inequalities: for any measurable event $A \in \mathcal{F}_{T}$ we have

$$
\begin{aligned}
E^{\mathbb{Q}}\left(\frac{1}{T} \int_{0}^{T} v_{t} d t-K\right)_{+} & \geq E^{\mathbb{Q}}\left[\left(\frac{1}{T} \int_{0}^{T} v_{t} d t-K\right)_{+} \cdot 1_{A}\right] \\
& \geq E^{\mathbb{Q}}\left[\left(\frac{1}{T} \int_{0}^{T} v_{t} d t-K\right) \cdot 1_{A}\right] \\
& =\frac{1}{T} \int_{0}^{T} E^{\mathbb{Q}}\left[\left(v_{t}-K\right) \cdot 1_{A}\right] d t .
\end{aligned}
$$

Next, we take events $A \in \mathcal{F}_{T}$ of the form $A=\{Z>\zeta\}$ with $\zeta \in \mathbb{R}$ a constant and

$$
Z=\frac{\int_{0}^{T} Z_{t} d t}{\sqrt{\operatorname{Var} \int_{0}^{T} Z_{t} d t}} \sim N(0,1)
$$

where $Z_{t}$ is the zero-mean Gaussian-OU process defined by equation (5). To search for $\zeta \in \mathbb{R}$ which yields the highest lower bound in (8), we have to solve the optimization problem

$$
\max _{\zeta \in \mathbb{R}} f(\zeta)=\frac{1}{T} \int_{0}^{T} E^{\mathbb{Q}}\left[\left(v_{t}-K\right) \cdot 1_{\{Z>\zeta\}}\right] d t .
$$

Letting $g(t, \zeta)=E^{\mathbb{Q}}\left[\left(v_{t}-K\right) \cdot 1_{\{Z>\zeta\}}\right]$ denote the integrand in the expression of $f(\cdot)$ above, we begin by noting the identity below, which follows from the law of total probability:

$$
g(t, \zeta)=\int_{\zeta}^{\infty} E^{\mathbb{Q}}\left(v_{t}-K \mid Z=z\right) \cdot \phi(z) d z
$$

where $\phi(\cdot)$ is the standard normal density. This allows us to easily see that

$$
\frac{\partial g}{\partial \zeta}(t, \zeta)=-E^{\mathbb{Q}}\left(v_{t}-K \mid Z=\zeta\right) \cdot \phi(\zeta) .
$$

It follows that the necessary maximum condition on $\zeta$ becomes

$$
f^{\prime}(\zeta)=\frac{1}{T} \int_{0}^{T} \frac{\partial g}{\partial \zeta}(t, \zeta) d t=-\frac{\phi(\zeta)}{T} \int_{0}^{T} E^{\mathbb{Q}}\left(v_{t}-K \mid Z=\zeta\right) d t=0
$$

or, equivalently

$$
\int_{0}^{T} E^{\mathbb{Q}}\left(v_{t} \mid Z=\zeta\right) d t=K T
$$


where, we recall that $v_{t}=\exp \left(\bar{y}(t)+Z_{t}\right)$. Next, we want to rewrite equation (9) for $\zeta$ in a more explicit way. Computing the conditional expectations $E^{\mathbb{Q}}\left(\exp \left(\bar{y}(t)+Z_{t}\right) \mid Z=\zeta\right)$ is straightforward using the properties of the Gaussian-OU process $Z_{t}$. In particular, we have that, for each $t \in[0, T]$, the pair $\left(Z_{t}, Z\right)$ is jointly normally distributed. Their covariance can be calculated explicitly to give

$$
\gamma(t)=\operatorname{Cov}\left(Z_{t}, Z\right)=\frac{\frac{\epsilon}{2 k}\left(2-e^{-k(T-t)}+e^{-k(T+t)}-2 e^{-k t}\right)}{\sqrt{T-\frac{3-4 e^{-k T+e^{-2 k T}}}{2 k}}} .
$$

Denoting the variance of $Z_{t}$ by

$$
\nu(t)=\operatorname{Var}\left(Z_{t}\right)=\frac{\epsilon^{2}}{2 k}\left(1-e^{-2 k t}\right)
$$

we have that the conditional distribution of $Z_{t}$ given $\{Z=\zeta\}$ is normal, with parameters

$$
Z_{t} \mid\{Z=\zeta\} \sim N\left(\gamma(t) \cdot \zeta, \nu(t)-\gamma(t)^{2}\right)
$$

which allows us to write (9) more explicitly and arrive at the necessary maximum condition for $\zeta$ as

$$
\int_{0}^{T} \exp \left(\bar{y}(t)+\gamma(t) \cdot \zeta+\frac{1}{2}\left(\nu(t)-\gamma(t)^{2}\right)\right) d t=K T .
$$

Since the left-hand side of (12) strictly increases from 0 to $\infty$ as $\zeta$ goes from $-\infty$ to $\infty$, we see that this equation has a unique solution $\zeta^{*} \in \mathbb{R}$. It is easy to check that $f^{\prime \prime}(\zeta)<0, \zeta \in \mathbb{R}$, and thus we conclude that $\zeta^{*}$ is our desired global maximum; in practice, the solution $\zeta^{*}$ is easily determined numerically, for example by applying Newton's root search algorithm to (12). Finally, to obtain the lower bound we have to compute the original integral from (8)

$$
L B=\frac{e^{-r T}}{T} \int_{0}^{T} E^{\mathbb{Q}}\left(\left(e^{\bar{y}(t)+Z_{t}}-K\right) \cdot 1_{Z-\zeta^{*}>0}\right) d t .
$$

After carrying out the remaining algebraic calculations (included in Appendix A.2), we can summarize our lower bound result in the following proposition.

Proposition 3.1. In the general Log-OU stochastic volatility model (1)(2), a call option on realized variance with variance strike $K$ and maturity $T$ satisfies the lower bound

$$
C_{v a r}(K) \geq L B=\frac{e^{-r T}}{T}\left[\int_{0}^{T} e^{\bar{y}(t)+\frac{1}{2} \nu(t)} \cdot N\left(-\zeta^{*}+\gamma(t)\right) d t-K \cdot N\left(-\zeta^{*}\right)\right]
$$


where $\zeta^{*}$ is the unique solution to the equation

$$
\int_{0}^{T} \exp \left(\bar{y}(t)+\gamma(t) \cdot \zeta+\frac{1}{2}\left(\nu(t)-\gamma(t)^{2}\right)\right) d t=K T
$$

with $\gamma(t)$ and $\nu(t)$ as defined in (10) and (11).

Before illustrating the numerical performance of this lower bound, we turn to the problem of deriving an upper bound. It will turn out that the upper bound is less sharp than the lower bound and hence less useful in practice. Nevertheless, the argument is interesting and we include it in our treatment for completeness.

Let $f_{t}$ be any integrable stochastic process such that $\int_{0}^{T} f_{t} d t=1$. We start with the following simple inequality:

$$
\begin{aligned}
E^{\mathbb{Q}}\left(\frac{1}{T} \int_{0}^{T} v_{t} d t-K\right)_{+} & =\frac{1}{T} E^{\mathbb{Q}}\left(\int_{0}^{T}\left(v_{t}-K T \cdot f_{t}\right) d t\right)_{+} \\
& \leq \frac{1}{T} \int_{0}^{T} E^{\mathbb{Q}}\left(v_{t}-K T \cdot f_{t}\right)_{+} d t .
\end{aligned}
$$

Next, similar to the idea in Thompson (1999), we take $f_{t}$ to have the particular form

$$
f_{t}=u_{t}+Z_{t}-\frac{1}{T} \int_{0}^{T} Z_{t} d t
$$

where $u \in C[0, T]$ is a deterministic, continuous function on $[0, T]$ such that $\int_{0}^{T} u_{t} d t=1$ and $Z_{t}$ the zero-mean Gaussian-OU process defined by (5). (Note that this choice of $f_{t}$ clearly satisfies the condition $\int_{0}^{T} f_{t} d t=1$.) To find the deterministic function $u \in C[0, T]$ which yields the lowest upper bound, we want to solve the problem

$$
\left\{\begin{array}{l}
\min _{u \in C[0, T]} \frac{1}{T} \int_{0}^{T} E^{\mathbb{Q}}\left(v_{t}-K T \cdot\left(u_{t}+X_{t}\right)\right)_{+} d t \\
\text { with } \int_{0}^{T} u_{t} d t=1
\end{array}\right.
$$

where we denoted $X_{t} \triangleq Z_{t}-\frac{1}{T} \int_{0}^{T} Z_{t} d t$. This problem can be formulated and solved as a simple problem in the calculus of variations. Let us first define $\psi(t, x):[0, T] \times \mathbb{R} \rightarrow \mathbb{R}$ as

$$
\psi(t, x)=E^{\mathbb{Q}}\left(v_{t}-K T \cdot\left(x+X_{t}\right)\right)_{+}
$$

and observe that we have

$$
\begin{aligned}
\frac{\partial \psi}{\partial x}(t, x) & =-K T \cdot P\left(v_{t}-K T \cdot X_{t} \geq K T \cdot x\right) \\
\frac{\partial^{2} \psi}{\partial x^{2}}(t, x) & \geq 0 .
\end{aligned}
$$


We now introduce the functional $F: C[0, T] \rightarrow \mathbb{R}$ associated with our constrained minimization problem:

$$
F(u)=\frac{1}{T} \int_{0}^{T} E^{\mathbb{Q}}\left(v_{t}-K T \cdot\left(u_{t}+X_{t}\right)\right)_{+} d t+\lambda\left(\int_{0}^{T} u_{t} d t-1\right)
$$

with $\lambda \in \mathbb{R}$ a Lagrange multiplier. It can be written more compactly in terms of $\psi(t, x)$ defined earlier

$$
\begin{aligned}
F(u) & =\frac{1}{T} \int_{0}^{T} \psi\left(t, u_{t}\right) d t+\lambda\left(\int_{0}^{T} u_{t} d t-1\right) \\
& =\frac{1}{T}\left[\int_{0}^{T}\left(\psi\left(t, u_{t}\right)+\lambda T \cdot u_{t}-\lambda\right) d t\right] .
\end{aligned}
$$

We calculate the first and second variations of the functional $F$ at $u$ to obtain

$$
\begin{aligned}
\delta F(u)(h) & =\frac{1}{T} \int_{0}^{T}\left(\frac{\partial \psi}{\partial x}\left(t, u_{t}\right)+\lambda T\right) \cdot h_{t} d t \\
\delta^{2} F(u)(h) & =\frac{1}{T} \int_{0}^{T} \frac{\partial^{2} \psi}{\partial x^{2}}\left(t, u_{t}\right) \cdot h_{t}^{2} d t
\end{aligned}
$$

where $h \in C[0, T]$ is any test function. Noting that the second variation is non-negative, we set the first variation equal to zero. From $\delta F(u)(h)=0$ for all $h \in C[0, T]$ we obtain the optimality condition, namely, for each $t \in[0, T]$ we must have

$$
\frac{\partial \psi}{\partial x}\left(t, u_{t}\right)+\lambda T=0
$$

or, from (16)

$$
K \cdot P\left(v_{t}-K T \cdot X_{t} \geq K T \cdot u_{t}\right)=\lambda .
$$

Upon writing out $v_{t}$ and $X_{t}$, explicitly this optimality condition reads

$$
K \cdot P\left(e^{\bar{y}(t)+Z_{t}}-K T \cdot\left(Z_{t}-\frac{1}{T} \int_{0}^{T} Z_{t} d t\right) \geq K T \cdot u_{t}\right)=\lambda .
$$

Because this expression combines log-normal and normal distributions, writing out an explicit solution for the optimal $u_{t}$ is not possible. Following Thompson (1999), by replacing $e^{Z_{t}}$ with $1+Z_{t}$ it becomes possible to determine a solution $u_{t}$ explicitly. Note from inequalities (13) that this, too, will provide us with an upper bound for the variance call option, although not the tightest one. Its performance will be illustrated numerically. The problem for $u \in C[0, T]$ now becomes

$$
K \cdot P\left(e^{\bar{y}(t)}\left(1+Z_{t}\right)-K T \cdot\left(Z_{t}-\frac{1}{T} \int_{0}^{T} Z_{t} d t\right) \geq K T \cdot u_{t}\right)=\lambda
$$


for all $t \in[0, T]$ and such that $\int_{0}^{T} u_{t} d t=1$. Once $u_{t}$ has been determined (and, hence, also $f_{t}$ in (13), (14)), the upper bound requires the computation of

$$
U B=\frac{e^{-r T}}{T} \cdot \int_{0}^{T} E^{\mathbb{Q}}\left(v_{t}-K T \cdot f_{t}\right)_{+} d t .
$$

Leaving the remaining algebraic calculations for the Appendix A.3, we finally obtain the upper bound as stated in the following proposition.

Proposition 3.2. In the general Log-OU stochastic volatility model (1)(2), a call option on realized variance with variance strike $K$ and maturity $T$ satisfies the upper bound

$$
\begin{gathered}
C_{\text {var }}(K) \leq U B=\frac{e^{-r T}}{T} \int_{0}^{T} \int_{-\infty}^{\infty} \phi(x) \cdot\left[\alpha(t, x \sqrt{\nu(t)}) N\left(\frac{\alpha(t, x \sqrt{\nu(t)})}{\beta(t)}\right)+\right. \\
\left.+\beta(t) \phi\left(\frac{\alpha(t, x \sqrt{\nu(t)})}{\beta(t)}\right)\right] d x d t
\end{gathered}
$$

where

$$
\nu(t)=\operatorname{Var}\left(Z_{t}\right)=\frac{\epsilon^{2}}{2 k}\left(1-e^{-2 k t}\right)
$$

and $\alpha(t, x), \beta(t)$ as given in Appendix A.3.

Table 1: Performance of Lower and Upper Bounds from Proposition 3.1 and Proposition 3.2; variance call prices expressed as implied volatilities-ofvolatility (\%).

\begin{tabular}{|c|rcc|ccc|ccc|}
\hline & \multicolumn{3}{|c|}{3 MonTHS } & \multicolumn{3}{c|}{6 MonTHS } & \multicolumn{3}{c|}{ 1 YEAR } \\
STRIKe & LB & Price & UB & LB & Price & UB & LB & Price & UB \\
\hline \hline 15 & 52.66 & 53.25 & 60.08 & 46.18 & 47.58 & 50.56 & 35.80 & 36.95 & 40.22 \\
20 & 55.56 & 55.81 & 61.48 & 48.32 & 48.94 & 51.36 & 37.09 & 37.74 & 39.90 \\
25 & 57.36 & 57.65 & 63.03 & 49.64 & 50.22 & 52.79 & 37.90 & 38.52 & 41.25 \\
30 & 58.62 & 59.10 & 64.60 & 50.56 & 51.16 & 54.15 & 38.47 & 39.17 & 43.11 \\
35 & 59.56 & 60.23 & 66.78 & 51.23 & 51.91 & 56.23 & 38.88 & 39.74 & 45.23 \\
40 & 60.28 & 61.08 & 69.42 & 51.76 & 52.51 & 58.59 & 39.21 & 40.19 & 47.31 \\
45 & 60.87 & 61.74 & 72.27 & 52.18 & 53.06 & 60.27 & 39.47 & 40.60 & 49.67 \\
\hline
\end{tabular}

Table (1) and Figure (2) show the performance of the lower and upper bounds of Propositions 3.1 and $3.2^{1}$, against the actual variance call prices

\footnotetext{
${ }^{1}$ Note: The one-dimensional integration required for the computation of the lower bound was carried out using a simple trapezoidal rule with time-step equal to a quarter of a day $\frac{1}{4} \cdot \frac{1}{252}$. The upper bound is more computationally expensive as it requires the computation of a double integral. This was carried out using an adaptive Simpson quadrature, as available in the Matlab package.
} 

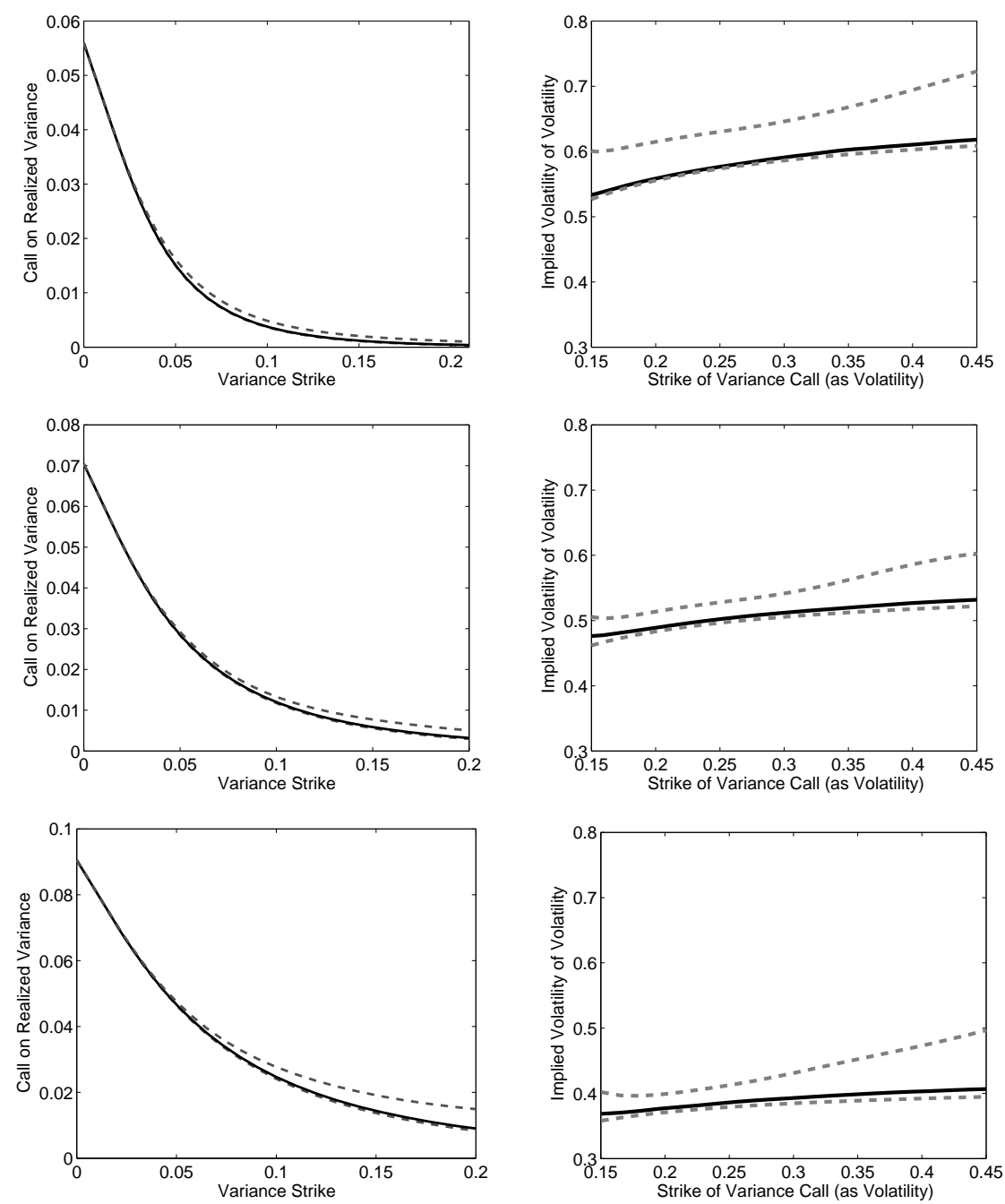

Figure 2: Solid black: variance call prices in Scott's (1987) model (obtained by Monte-Carlo with geometric control variates), Dashed gray: Lower and Upper bounds from Propositions 3.1 and 3.2 : absolute prices (Left), implied volatilities-of-volatility (Right). Maturities: 3 months (top), 6 month (middle), 1 year (bottom). 
computed by Monte Carlo, with geometric control variates. In Figure (2), we plot both the absolute prices (in the left panel) and the corresponding log-normal implied volatilities of volatility (in the right panel). Across a wide range of volatility strikes (ranging from 0.15 to 0.45 ) and across maturities (3 months, 6 months and 1 year), we notice that, the lower bound remains very sharp. More precisely, the difference between the implied volatility-ofvolatility corresponding to the Monte Carlo price and the one corresponding to the lower bound is roughly of the order of one volatility-of-volatility point - which is well within any reasonable bid-offer spread for such volatility products. The upper bound is less sharp and, hence, will be of less practical interest.

\section{Further Applications}

In this section we present two additional issues, which are of particular interest in practice ${ }^{2}$, and we discuss the application of the results obtained in the previous sections.

In order to obtain a more flexible volatility-of-volatility term structure, it may be necessary to consider a variance process driven by multiple factors. For example, an important special case is a two-factor model, combining a fast mean-reverting factor and a slow mean-reverting factor, thus allowing control of both the short-end and the long-end of the volatility-of-volatility term structure. Specifically, assume that the logarithm of instantaneous variance $y_{t}$ is now given by:

$$
y_{t}=\bar{y}(t)+Z_{t}^{(1)}+Z_{t}^{(2)}
$$

where $Z_{t}^{(1)}$ and $Z_{t}^{(2)}$ are - similar to $Z_{t}$ in $(5)$ - zero-mean Gaussian-OU processes driven by two (correlated) Brownian motions $W_{t}^{(1)}$ and $W_{t}^{(2)}$.

Importantly, we remark that the proofs of both Proposition 3.1 and Proposition 3.2 depend only on the key assumption that the log of instantaneous variance can be written as

$$
y_{t}=\bar{y}(t)+Z_{t}
$$

where $\bar{y}(t)$ is a deterministic function and $Z_{t}$ is a Gaussian process; in particular, we can take the Gaussian process $Z_{t}$ as $Z_{t}=Z_{t}^{(1)}+Z_{t}^{(2)}$. Now, the lower bound in the statement of Proposition 3.1 applies ad-litteram to the new setup, provided we update the functions $\nu(t)$ and $\gamma(t)$ to reflect the covariance structure of the new Gaussian process $Z_{t}$. The same applies to Proposition 3.2 by properly updating the functions $\alpha(t, x)$ and $\beta(t)$.

\footnotetext{
${ }^{2}$ We thank an anonymous referee for drawing our attention to the topics treated in this section.
} 
The second issue we address refers to the discrete sampling of variance. In practice, options on realized variance are usually specified with discrete daily sampling; the discretization effect becomes particularly important for shorter expiries, for example, less than 6 months. A fast technique to account for discrete sampling, based on Fourier inversion, was first developed and proposed by Sepp (2011). Subsequently, an alternative approach, which applies in general stochastic volatility models, was obtained in Drimus, Farkas (2011). Since, under Log-OU dynamics, the Fourier transform of realized variance is not available in closed-form, we now show a simple application of the latter method to the lower bound of Proposition 3.1. Drimus, Farkas (2011) obtain the following simple discretization adjustment:

$$
C_{n}(K)-C(K)=\frac{K^{2}}{n} \cdot q(K)+O\left(\frac{1}{n^{2}}\right)
$$

where $C_{n}(K), C(K)$ denote the prices of call options on discretely and continuously sampled variance, $q(K)$ is the density of continuously sampled variance and $n$ is the number of sampling points. By the classic result of Breeden, Litzenberger (1978), the density $q(K)$ can be recovered from the call prices by $q(K)=e^{r T} \cdot \frac{\partial^{2} C}{\partial K^{2}}(K)$. After computing the lower bound prices $C^{\mathrm{LB}}(K)$ of Proposition 3.1 for a sequence of variance strikes $K_{1}, K_{2}, \ldots$ with $\Delta K=K_{i}-K_{i-1}$, we propose the correction for discrete sampling as

$$
C^{\mathrm{LB}}\left(K_{i}\right)+\frac{K_{i}^{2}}{n} \cdot e^{r T} \cdot \frac{C^{\mathrm{LB}}\left(K_{i}-\Delta K\right)-2 \cdot C^{\mathrm{LB}}\left(K_{i}\right)+C^{\mathrm{LB}}\left(K_{i}+\Delta K\right)}{\Delta K^{2}}
$$

where we have applied a centered finite difference quotient to approximate the second derivative in strike. Of course, it cannot be guaranteed that the corrected prices will also act as a lower bound for options on discretely sampled variance, but we found that it will capture most of the discretization effect. Using the numerical example of the previous section, we show in Figure (3) the discretization correction applied to the lower bound of Proposition 3.1, against prices of options on discrete variance computed by Monte Carlo, for a maturity of 3 months. Across variance strikes, the average error between the proposed correction and actual prices has been less than one volatility-of-volatility point.

\section{Conclusions}

We have introduced a general class of Log-OU stochastic volatility models. This class of models offers a significant improvement over the standard Heston (1993) model by generating a more acceptable implied volatilityof-volatility skew. In this context, the valuation problem for options on realized variance bears certain analogies to Asian options. The main difference is that the driving process is now a mean-reverting OU process, as 


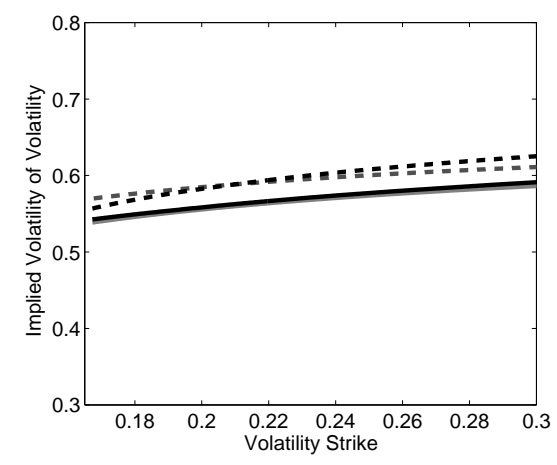

Figure 3: Solid: variance call prices with continuous sampling (black) and lower bound from Proposition 3.1 (gray), Dashed: variance call prices with discrete sampling (black) and the discretization correction in (17) (gray). Maturity of 3 months.

opposed to a standard Brownian motion. We show how to extend a number of effective Asian option methods to the pricing of options on realized variance and obtain, in particular, a very tight lower bound. In practice, the error from using the lower bound will be smaller than the usual bid-offer spread for such volatility products. For completeness, we also derived an upper bound. Finally, we have shown how the results can be applied to accommodate multiple factors and discrete sampling of realized variance.

\section{Appendix}

A.1. A Black-Scholes style formula is obtained for the geometric control variate of a call option on realized variance as follows. Specifically, to value

$$
C_{\text {var }}^{\text {Geom. }}(K)=e^{-r T} E^{\mathbb{Q}}\left(\exp \left(\frac{1}{T} \int_{0}^{T}\left(\bar{y}(t)+Z_{t}\right) d t\right)-K\right)_{+}
$$

we use that

$$
\frac{1}{T} \int_{0}^{T}\left(\bar{y}(t)+Z_{t}\right) d t \sim N\left(\frac{1}{T} \int_{0}^{T} \bar{y}(t) d t, \frac{\epsilon^{2}}{T^{2} k^{2}}\left(T-\frac{3-4 e^{-k T}+e^{-2 k T}}{2 k}\right)\right) .
$$

In Scott's (1987), and respectively Bergomi's (2005), models we have

$$
\begin{aligned}
\frac{1}{T} \int_{0}^{T} \bar{y}(t) d t= & \log (\theta)+\left(y_{0}-\log (\theta)\right) \cdot \frac{1-e^{-k T}}{k T} \\
\frac{1}{T} \int_{0}^{T} \bar{y}(t) d t= & y_{0} \cdot \frac{1-e^{-k T}}{k T}+\frac{1}{T} \int_{0}^{T} \log \left(\theta_{u}\right) \cdot\left(1-e^{-k(T-u)}\right) d u \\
& -\frac{\epsilon^{2}}{4 k}\left(1-\frac{1-e^{-2 k T}}{2 k T}\right) .
\end{aligned}
$$


Denoting $m=\frac{1}{T} \int_{0}^{T} \bar{y}(t) d t$ and $v^{2}=\operatorname{Var}\left(\frac{1}{T} \int_{0}^{T}\left(\bar{y}(t)+Z_{t}\right) d t\right)$ we obtain the Black-Scholes style formula for the control variate

$$
C_{\text {var }}^{\text {Geom. }}(K)=e^{-r T}\left[e^{m+\frac{v^{2}}{2}} \cdot N\left(d_{1}\right)-K \cdot N\left(d_{2}\right)\right]
$$

with

$$
\begin{aligned}
d_{1} & =\frac{\log \left(\frac{e^{m+\frac{v^{2}}{2}}}{K}\right)+\frac{v^{2}}{2}}{v} \\
d_{2}= & d_{1}-v .
\end{aligned}
$$

A.2. To calculate the inner expectation appearing in the expression of the lower bound

$$
L B=\frac{e^{-r T}}{T} \int_{0}^{T} E\left(\left(e^{\bar{y}(t)+Z_{t}}-K\right) \cdot 1_{Z-\zeta^{*}>0}\right) d t
$$

we use the following simple identity given in Thompson (1999):

$$
E\left(\left(e^{X}-K\right) \cdot 1_{Y>0}\right)=e^{\mu_{X}+\frac{1}{2} \sigma_{X}^{2}} \cdot N\left(\frac{\mu_{Y}+\sigma_{X Y}}{\sigma_{Y}}\right)-K \cdot N\left(\frac{\mu_{Y}}{\sigma_{Y}}\right)
$$

which holds for any bivariate normal vector $(X, Y)$ with mean $\left(\mu_{X}, \mu_{Y}\right)$ and covariance matrix

$$
\left(\begin{array}{cc}
\sigma_{X}^{2} & \sigma_{X Y} \\
\sigma_{X Y} & \sigma_{Y}^{2}
\end{array}\right)
$$

Writing $v_{t}=e^{\bar{y}(t)+Z_{t}}$, we set $X=\bar{y}(t)+Z_{t}, Y=Z-\zeta^{*}$ with $\mu_{X}=\bar{y}(t)$, $\mu_{Y}=-\zeta^{*}, \sigma_{X}^{2}=\nu(t)=\frac{\epsilon^{2}}{2 k}\left(1-e^{-2 k t}\right), \sigma_{Y}^{2}=1$ and covariance

$$
\sigma_{X Y}=\frac{\frac{\epsilon}{2 k}\left(2-e^{-k(T-t)}+e^{-k(T+t)}-2 e^{-k t}\right)}{\sqrt{T-\frac{3-4 e^{-k T+e^{-2 k T}}}{2 k}}} .
$$

as was given in the main text and denoted there $\gamma(t)$. Finally, plugging these terms in the identity above we obtain

$$
L B=\frac{e^{-r T}}{T} \int_{0}^{T}\left[e^{\bar{y}(t)+\frac{1}{2} \nu(t)} \cdot N\left(-\zeta^{*}+\gamma(t)\right)-K \cdot N\left(-\zeta^{*}\right)\right] d t .
$$

A.3. Recall that we need to determine $u \in C[0, T]$ such that

$$
K \cdot P\left(e^{\bar{y}(t)}\left(1+Z_{t}\right)-K T \cdot\left(Z_{t}-\frac{1}{T} \int_{0}^{T} Z_{t} d t\right) \geq K T \cdot u_{t}\right)=\lambda
$$

for all $t \in[0, T]$, or equivalently

$$
K \cdot P\left(\left(e^{\bar{y}(t)}-K T\right) \cdot Z_{t}+K \int_{0}^{T} Z_{t} d t+e^{\bar{y}(t)} \geq K T \cdot u_{t}\right)=\lambda .
$$


Letting

$$
N_{t}=\left(e^{\bar{y}(t)}-K T\right) \cdot Z_{t}+K \int_{0}^{T} Z_{t} d t+e^{\bar{y}(t)}
$$

we first observe that, since $\left(Z_{t}, \int_{0}^{T} Z_{t} d t\right)$ are jointly normally distributed, it follows that $N_{t}$ is also normal with

$$
N_{t} \sim N\left(e^{\bar{y}(t)}, \omega(t)\right)
$$

where we denote $\operatorname{Var}\left(N_{t}\right)=\omega(t)$. Note that

$$
\begin{aligned}
\omega(t)= & \left(e^{\bar{y}(t)}-K T\right)^{2} \cdot \operatorname{Var}\left(Z_{t}\right)+K^{2} \cdot \operatorname{Var}\left(\int_{0}^{T} Z_{t} d t\right)+ \\
& +2 K\left(e^{\bar{y}(t)}-K T\right) \cdot \operatorname{Cov}\left(Z_{t}, \int_{0}^{T} Z_{t} d t\right)
\end{aligned}
$$

with

$$
\begin{aligned}
\operatorname{Var}\left(Z_{t}\right) & =\frac{\epsilon^{2}}{2 k}\left(1-e^{-2 k t}\right) \\
\operatorname{Var}\left(\int_{0}^{T} Z_{t} d t\right) & =\frac{\epsilon^{2}}{k^{2}}\left(T-\frac{3-4 e^{-k T}+e^{-2 k T}}{2 k}\right) \\
\operatorname{Cov}\left(Z_{t}, \int_{0}^{T} Z_{t} d t\right) & =\frac{\epsilon^{2}}{2 k^{2}}\left(2-e^{-k(T-t)}+e^{-k(T+t)}-2 e^{-k t}\right) .
\end{aligned}
$$

Written in terms of $N_{t}$ the condition for $u_{t}$ becomes

$$
P\left(N_{t} \geq K T \cdot u_{t}\right)=\frac{\lambda}{K}
$$

which is equivalent to

$$
\frac{K T \cdot u_{t}-e^{\bar{y}(t)}}{\sqrt{\omega(t)}}=\gamma
$$

where we denote $N^{-1}\left(1-\frac{\lambda}{K}\right)=\gamma$. Solving for $u_{t}$ gives

$$
u_{t}=\frac{1}{K T} \cdot\left(e^{\bar{y}(t)}+\gamma \sqrt{\omega(t)}\right)
$$

To find the constant $\gamma$ we impose the condition $\int_{0}^{T} u_{t} d t=1$ which gives

$$
\gamma=\frac{K T-\int_{0}^{T} e^{\bar{y}(t)} d t}{\int_{0}^{T} \sqrt{\omega(t)}} .
$$

Finally, having determined $u_{t}$, to calculate the upper bound we must compute

$$
U B=\frac{e^{-r T}}{T} \int_{0}^{T} E\left(e^{\bar{y}(t)+Z_{t}}-K T \cdot\left(u_{t}+Z_{t}-\frac{1}{T} \int_{0}^{T} Z_{t} d t\right)\right)_{+} .
$$


For the inner expectation, we proceed by conditioning on $Z_{t}$ and using that

$$
\int_{0}^{T} Z_{t} d t \mid\left\{Z_{t}=z\right\} \sim N\left(\frac{\operatorname{Cov}\left(Z_{t}, \int_{0}^{T} Z_{t} d t\right)}{\operatorname{Var}\left(Z_{t}\right)} \cdot z, \operatorname{Var}\left(\int_{0}^{T} Z_{t} d t \mid Z_{t}\right)\right)
$$

where

$$
\operatorname{Var}\left(\int_{0}^{T} Z_{t} d t \mid Z_{t}\right)=\operatorname{Var} \int_{0}^{T} Z_{t} d t-\frac{\operatorname{Cov}^{2}\left(Z_{t}, \int_{0}^{T} Z_{t} d t\right)}{\operatorname{Var} Z_{t}}
$$

and where the expressions of the variances and covariances are given explicitly in (17),(18) and (19). Integrating out with respect to the density of $Z_{t}$, we write the inner expectation as

$$
\int_{-\infty}^{\infty} \frac{1}{\sqrt{\nu(t)}} \phi\left(\frac{z}{\sqrt{\nu(t)}}\right) \cdot E(\beta(t) \cdot N(0,1)+\alpha(t, z))_{+} d z
$$

where

$$
\begin{gathered}
\beta(t)=K \sqrt{\operatorname{Var}\left(\int_{0}^{T} Z_{t} d t \mid Z_{t}\right)} \\
\alpha(t, z)=e^{\bar{y}(t)+z}-K T \cdot\left(u_{t}+z\right)+K \cdot \frac{\operatorname{Cov}\left(Z_{t}, \int_{0}^{T} Z_{t} d t\right)}{\operatorname{Var} Z_{t}} \cdot z .
\end{gathered}
$$

Finally, using the basic identity from Thomson (1999)

$$
E(b \cdot N(0,1)+a)_{+}=a \cdot N\left(\frac{a}{b}\right)+b \cdot \phi\left(\frac{a}{b}\right)
$$

$a \in \mathbb{R}, b \in \mathbb{R}$, we obtain the expression for the upper bound

$\frac{e^{-r T}}{T} \int_{0}^{T} \int_{-\infty}^{\infty} \frac{1}{\sqrt{\nu(t)}} \phi\left(\frac{z}{\sqrt{\nu(t)}}\right) \cdot\left[\alpha(t, z) \cdot N\left(\frac{\alpha(t, z)}{\beta(t)}\right)+\beta(t) \cdot \phi\left(\frac{\alpha(t, z)}{\beta(t)}\right)\right] d z d t$.

Making the change of variable $z=x \cdot \sqrt{\nu(t)}$ we arrive at the final form

$$
\begin{gathered}
C_{v a r}(K) \leq U B=\frac{e^{-r T}}{T} \int_{0}^{T} \int_{-\infty}^{\infty} \phi(x) \cdot\left[\alpha(t, x \sqrt{\nu(t)}) N\left(\frac{\alpha(t, x \sqrt{\nu(t)})}{\beta(t)}\right)+\right. \\
\left.+\beta(t) \phi\left(\frac{\alpha(t, x \sqrt{\nu(t)})}{\beta(t)}\right)\right] d x d t
\end{gathered}
$$




\section{References}

[1] Bergomi, L. (2005), Smile dynamics 2, Risk Magazine, October Issue, $67-73$.

[2] Breeden, D., Litzenberger, R. (1978), Prices of state contingent claims implicit in option prices, Journal of Business, 51(6), 621-651.

[3] Drimus, G., Farkas, W. (2011) Valuation of options on discretely sampled variance, preprint, Dept. of Mathematics, Univ. of Copenhagen, and Inst. of Banking and Finance, Univ. of Zürich.

[4] Gatheral, J. (2006), The volatility surface: A practitioner's guide, Wiley Finance.

[5] Heston, S. (1993), A closed-form solution for options with stochastic volatility with applications to bond and currency options, The Review of Financial Studies 6, 327-343.

[6] Kemma, A., Vorst, A. (1990), A pricing method for options based on average asset values, Journal of Banking and Finance, 14, 113-129.

[7] Lewis, A. (2000), Option valuation under stochastic volatility, Finance Press.

[8] Rogers, L.C.G, Shi, Z. (1995) The value of an Asian option, Journal of Applied Probability, 32, 1077-1088.

[9] Romano, M., Touzi, N., (1997) Contingent claims and market completeness in a stochastic volatility model, Mathematical Finance, Vol. 7, No. 4, 399-410.

[10] Sepp, A. (2008), Pricing options on realized variance in the Heston model with jumps in returns and volatility, Journal of Computational Finance, volume 11 / 4, 33-70.

[11] Sepp, A. (2011), Pricing options on realized variance in the Heston model with jumps in returns and volatility II: An approximate distribution of the discrete variance, forthcoming, Journal of Computational Finance.

[12] Scott, L. (1987), Option pricing when the variance changes randomly: theory, estimation and an application, Journal of Financial and Quantitative Analysis 22, 419-438.

[13] Thompson, G.W.P. (1999), Fast narrow bounds on the value of Asian options, working paper, Center for Financial Research, University of Cambridge. 